\title{
Common Coil Magnet Program at BNL
}

\author{
R. Gupta, M. Anerella, J. Cozzolino, J. Escallier, G. Ganetis, A. Ghosh, \\ M. Harrison, G. Morgan, J. Muratore, B. Parker, W. Sampson and P. Wanderer
}

\begin{abstract}
The goal of the common coil magnet R\&D program at Brookhaven National Laboratory (BNL) is to develop a $\mathbf{1 2 . 5}$ $T, 40 \mathrm{~mm}$ aperture dipole magnet using "React and Wind Technology" with High Temperature Superconductors (HTS) playing a major role. Due to its "conductor friendly" nature, the common coil design is attractive for building high field 2-in-1 dipoles with brittle materials such as $\mathrm{HTS}$ and $\mathrm{Nb}_{3} \mathrm{Sn}$. At the current rate of development, it is expected that a sufficient amount of HTS with the required performance would be available in a few years for building a short magnet. In the interim, the first generation dipoles will be built with $\mathrm{Nb}_{3} \mathrm{Sn}$ superconductor. They will use a "React and Wind" technology similar to that used in HTS and will produce a $12.5 \mathrm{~T}$ central field in a $40 \mathrm{~mm}$ aperture. The $\mathrm{Nb}_{3} \mathrm{Sn}$ coils and support structure of this magnet will become a part of the next generation hybrid magnet with inner coils made of HTS. To develop various aspects of the technology in a scientific and experimental manner, a 10-turn coil program has been started in parallel. The program allows a number of concepts to be evaluated with a rapid throughput in a cost-effective way. Three 10-turn $\mathrm{Nb}_{3} \mathrm{Sn}$ coils have been built and one HTS coil is under construction. The initial test results of this "React \& Wind" 10-turn coil program are presented. It is also shown that a common coil magnet design can produce a field quality that is as good as a conventional cosine theta design.
\end{abstract}

Index Terms-Accelerators, High Temperature Superconductors, Magnets, Superconducting Magnets.

\section{INTRODUCTION}

$\mathrm{T}$ HE common coil magnet design [1-4] is a 2-in-1 block design based on flat racetrack coils that are shared between two apertures (Fig. 1). The bend radius in the ends is much. larger than that in a conventional design as it is determined by the separation between the two apertures rather than the size of the aperture itself. The design offers a "conductor friendly" geometry that is suitable for brittle materials and for containing large Lorentz forces. In Phase I of this program, a proof of principle $6 \mathrm{~T}$, NbTi dipole was built and commissioned [5] at BNL. Magnets based on the common coil design have also been built at Lawrence Berkeley National Laboratory [6,7]. Another is in the advanced design stage at the Fermi National Accelerator Laboratory [8]. The Phase I dipole at BNL now serves as a background field test facility for HTS coils in a hybrid common coil magnet geometry. Phase II of this program uses more engineering resources. The goal of the program is to

Manuscript received September 18, 2000.

This work was supported by the U.S. Department of Energy under contract No. DE-AC02-98CH10886.

The authors are with the Brookhaven National Laboratory, Upton, NY 11973, US $\Lambda$. (Email address of primary author: gupta@bnl.gov). gupta@bnl.gov: http://magnets.rhic.bnl.gov/staff/gupta. develop a magnet design and technology that can be eventually used by industry to carry out large scale magnet production and to reduce the overall cost of the machine. The possible applications of the common coil magnet program are a very large hadron collider (VLHC) and a RHIC upgrade. Other possible uses of racetrack HTS coil technology are magnets for muon colliders and magnets for upgrading the interaction regions of existing machines.

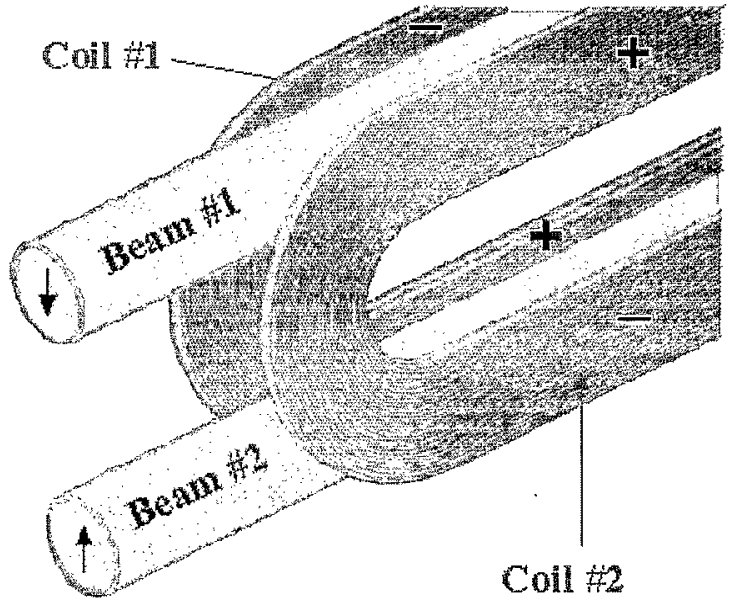

Fig. 1. The main coils of the "conductor friendly" common coil design concept.

\section{10-TURN COIL PROGRAM}

\section{A. Program Philosophy}

The philosophy behind the 10-turn coil program is to design a simple and yet flexible magnet R\&D program that provides a rapid throughput. After an initial setup, the fabrication of each coil uses only a small amount of additional resources both in terms of cost and time. Therefore one can afford to build many coils to systematically evaluate the impact of an individual process. One can even afford to sacrifice a few coils in the process of developing new technology and/or experimentally determining the limit of a particular approach. This program is complementary to the traditional magnet R\&D program where significant resources are committed to the development and construction of a magnet to ensure its good performance.

One 10-turn common coil test magnet (with only $0.25 \mathrm{~mm}$ coil spacing) has been built and tested in a short time and with limited resources. The techniques used for insulating the prereacted $\mathrm{Nb}_{3} \mathrm{Sn}$ cable and for winding coils were similar to those used in NbTi magnets. This is a significant departure from the conventional wisdom that brittle superconductors, such as $\mathrm{Nb}_{3} \mathrm{Sn}$ and HTS, should be handled very delicately. Therefore, a reasonable quench performance is of major practical consequence as it suggests that the matured $\mathrm{NbTi}$ 
technology can be adopted for making "conductor friendly" common coil magnets with brittle superconductors and for scaling up the magnet production.

\section{B. Design Parameters}

The minimum bend radius in the 10 -turn coil design is 70 $\mathrm{mm}$, the same as in the high field magnet design. The straight section is $300 \mathrm{~mm}$ and only $\sim 11$ meter of insulated cable is used in building a coil. The electrical and mechanical support structure has been designed to provide maximum praclical flexibility. It can handle from one to six coils allowing a maximum of $9 \mathrm{~T}$ field with almost no spacing between them. It also allows three pairs of coils to use three different currents. The power supply at BNL, can deliver up to $30 \mathrm{kA}$ of current.

The $\mathrm{Nb}_{3} \mathrm{Sn}$ cable for the first three coils was provided by Lawrence Berkeley National Laboratory (LBNL) and is similar to the cable used in magnet RD2 [6] except that the critical current is $20 \%$ lower, based on extracted strand measurements at the University of Twente [9]. The cable uses 30 strands of chrome-plated $0.8 \mathrm{~mm}$ diameter wire and has a nominal width of $12.3 \mathrm{~mm}$ and thickness of $1.45 \mathrm{~mm}$. The $\mathrm{Cu} / \mathrm{Sc}$ ratio is 1.5 and $J_{c}$ is only $\sim 600 \mathrm{~A} / \mathrm{mm}^{2}$ at $12 \mathrm{~T}$. The computed short sample, based on the measurements of a part of the cable at the BNL test facility is $\sim 10 \mathrm{kA}$. This produces a field of $\sim 4.6 \mathrm{~T}$ in a common coil configuration (Fig. 1) with $0.25 \mathrm{~mm}$ gap between two coils. However, the computed short sample is $\sim 9.4 \mathrm{kA}$ based on the cable degradation observed in RD2 magnet and cable measurements performed elsewhere by LBNL. The model calculations account for the fact that the coil separation (aperture) is comparable to the insulation between two turns.

\section{Coil Construction}

The $\mathrm{Nb}_{3} \mathrm{Sn}$ cable was reacted at $\mathrm{BNL}$. The cable was insulated with a double over-laying wrap of 75 micron fiberglass (150 microns on each side of cable) with the equipment and procedure that are used in insulating the $\mathrm{NbTi}$ cable. The pre-reacted $\mathrm{Nb}_{3} \mathrm{Sn}$ cable was wound on an iron bobbin with a low tension ( $\sim 10$ pounds or $\sim 50$ newtons) but otherwise using the same winder and procedure that are used in making standard NbTi coils. Clamps were used for holding the cable tight and in place during the winding. They were only partly successful, as some looseness was left between the turns.

The leads were stabilized with $\mathrm{NbTi}$. Nomex sheet was used to insulate the cable from the bobbin. The common coil design has the unique advantage that the splice to the internal lead can be made in a low field region, since there is adequate space in the middle of the coil where the field is low (Fig. 2).

\section{Coil Impregnation}

The coil wound on a bobbin, G-10 side plates, G-10 end saddles and $0.1 \mathrm{~mm}$ fiberglass cloth on two sides of a coil form a complete cassette. These parts are placed inside metal side and edge plates to form an impregnated module. O-rings are used to create a vacuum seal. Freekote is used as a mold

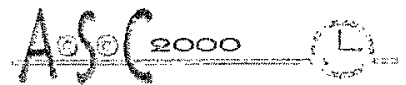

release in removing side plates and edge plates after the impregnation.

The primary epoxy used for impregnation is CTD101 [10]. Stycast 2850 [11] is used for filling large gaps. De-airing of the CTD101 was done at $60 \mathrm{C}$ and $\sim 1$ Torr in a glass container that was filled only to a few inches. However, the pressure in the mold was brought to $\sim 50$ Torr when the epoxy was injected. Since epoxy was de-aired at a much lower pressure, this procedure reduced the chance of any bubble remaining trapped inside the impregnated coil.

\section{E. Mechanical Support}

The Lorentz forces act inward when a single coil is tested or when many coils are tested with current flowing in the same direction. In the common coil configuration (Fig. 1), the Lorentz forces are mostly horizontal and outward towards the support structure. The support structure consists of parallel stainless steel plates around the coil cassette with bolts taking the outward pressure. The holes in the bobbin (Fig. 2) are used for providing additional support bolts.

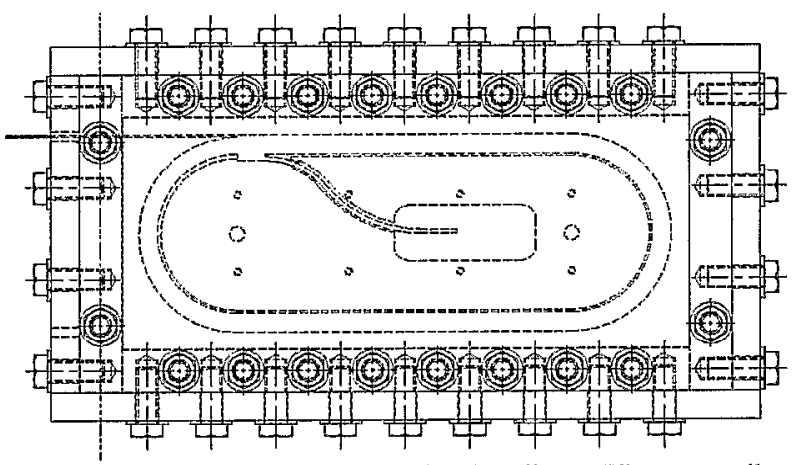

Fig. 2. Support structure for single and double coil test. When two coils are testcd in the common coil mode, additional bolts are put through the holes.

\section{F. Test Results}

Two tests have been performed in different configurations. In the first test a single coil was powered ten times to $9000 \mathrm{~A}$ (the power supply limit in this test setup) and no quench was observed. In the second test, the two coils were powered in the common coil configuration. Fig. 3 shows the quench plot of this just completed test. The magnet quickly reached plateau at $8240 \mathrm{~A}$ at a ramp rate of $50 \mathrm{~A} / \mathrm{sec}$. At this ramp rate the quench current is reduced by $\sim 5 \%$ as per the ramp rate studies in LBNL's RD2 magnet [6]. The change in quench current with temperature was also consistent with RD2. All quenches were in the same coil, had similar characteristics and seemed to be in the same location. The support structure and splices behaved well and the performance appeared to be conductor limited. Allowing for cable degradation similar to that in $\mathrm{RD} 2$ and ramp rate adjustments, the plateau was $\sim 8 \%$ below the computed short sample. However, the quench current might be $\sim 5 \%$ higher due to the uncertainty involved in the cstimate. As per the conventional wisdom this is a low degradation as the brittle $\mathrm{Nb}_{3} \mathrm{Sn}$ cable went through large strain during the insulation, winding and magnet construction process. 
The reasonable performance of the first magnet (one training quench, relatively small degradation) augurs well for the future of "React and Wind" common coil magnet technology. By the very nature of the 10-turn coil program, it should be possible to systematically find out the source of degradation. One can then either remove that by adjusting the process or parameters or may accept a part of it after determining a level of acceptance for an overall optimization of the cost and complexity of the construction process.

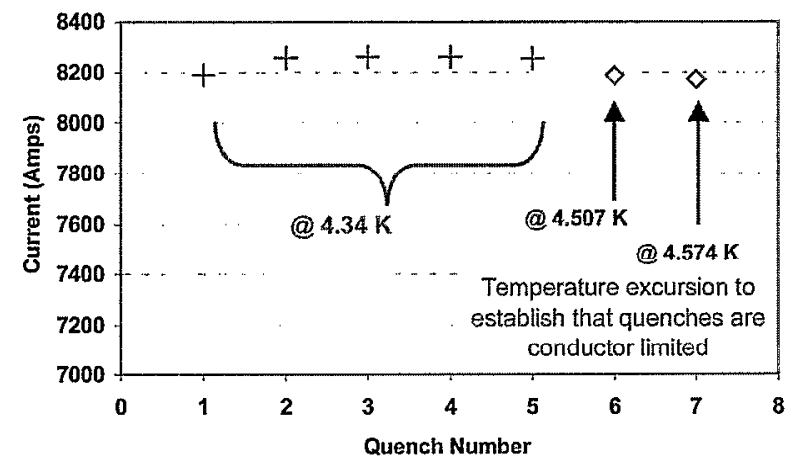

Fig. 3. Test results of the first 10 turn common coil magnet built with prereacted $\mathrm{Nb}_{3} \mathrm{Sn}$ conductor with almost no separation between the two coils.

\section{HIGII FIELD DESIGN}

The high field $\sim 12.5 \mathrm{~T}$ design is in a preliminary stage. The first order magnetic design is complete and the cable parameters are specified. As shown in Fig. 4, it is a two layer design with minimum amount of space between coils consumed by internal support structure. The iron outer radius is $250 \mathrm{~mm}$.

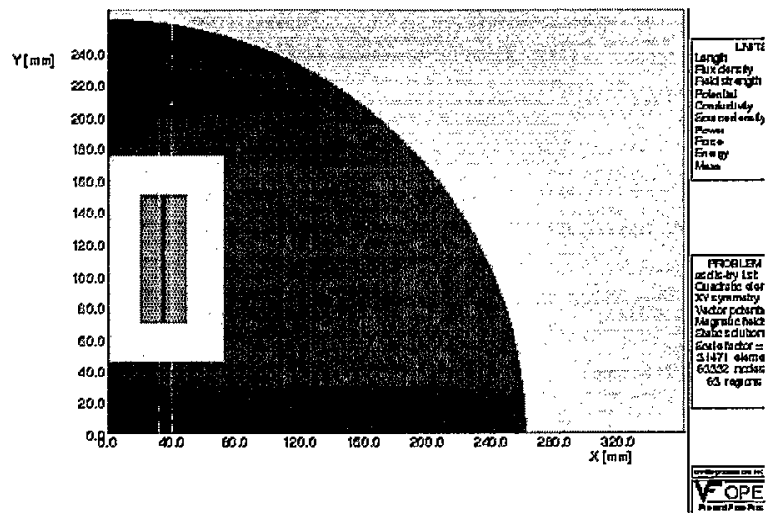

Fig. 4. A computer model of one quadrant (one-half of the two apertures) of the high field $40 \mathrm{~mm}$ aperture magnetic design.

Both inner and outer layers use 30 strand cable with a nominal bare width of $12.4 \mathrm{~mm}$ and thickness of $1.45 \mathrm{~mm}$. The nominal wire diameter is $0.8 \mathrm{~mm}$ and the expected $\mathrm{J}_{\mathrm{c}}$ is $2000 \mathrm{~A} / \mathrm{mm}^{2}$ at $12 \mathrm{~T}$ and $4.2 \mathrm{~K}$. The copper to superconductor ratio is 1 in the inner layer and 1.86 in the outer. For a $40 \mathrm{~mm}$ coil (and physical) aperture, the expected quench field is $\sim 12.5 \mathrm{~T}$. The current density in copper at quench is $1400-1500 \mathrm{~A} / \mathrm{mm}^{2}$. The grading between the two layers is achieved with two different power supplies, allowing the grading to be varied during a magnet test. This provides a

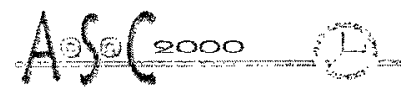

unique facility for studying various conductor-related issues in a "real magnet" situation. For example, a large change in current density in the copper (as much as $\sim 1000 \mathrm{~A} / \mathrm{mm}^{2}$ ) at quench can be obtained by changing the relative current between the two layers. This would be perhaps the first time that such a scientific study is carried out within the same magnet. This should provide useful guidance on this important parameter for the future magnet designs.

\section{HTS PROGRAM}

BNL is leading the efforts to include High Temperature Superconducting (HTS) coils in accelerator magnets. HTS continues to make rapid progress in performance. Now HTS wires carry more current at $15 \mathrm{~T}$ and $4.3 \mathrm{~K}$ than conventional low temperature superconductors of similar size. The first step was to wind and test a 1-meter long coil made with (BSCCO 2223) HTS tape [12]. Recently, BNL has also obtained three pieces of 18-strand cable ranging from 11 meters to 20 meters in length, each sufficient to make a 10 turn coil. In addition, about 30 meters of mixed strand cable ( 2 strands of BSCCO 2212 and 16 strands of silver) have also been obtained. These cables have been produced in collaboration with Intermagnetic General Corporation (IGC), Showa Electric (Japan) and LBNL.

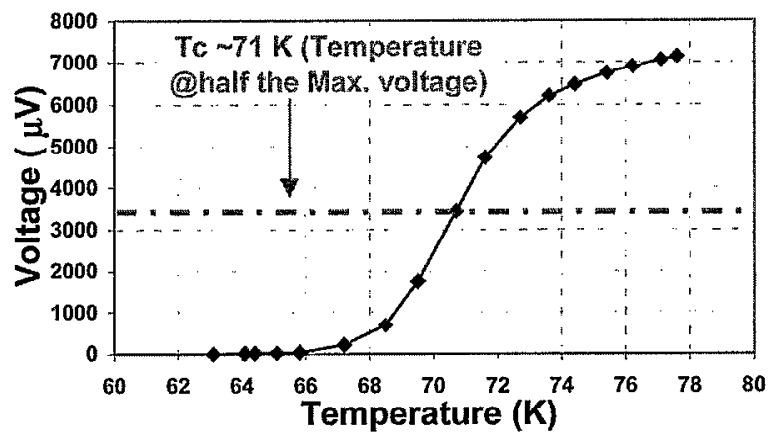

Fig. 5. Temperature dependence of the resistance (measured as a drop in voltage) for $1 \mathrm{~A}$ current in BSCCO cable. A transition from normal to superconducting phase can be seen.

Fig. 5 shows a transition from normal to superconducting phase when $1 \mathrm{~A}$ current was passed in $\sim 11$ meter of this cable in liquid nitrogen (LN2) at BNL. To obtain high performance in high field magnets, the HTS cable would be operated at $\sim 4$ $\mathrm{K}$ in liquid helium. However, the LN2 testing provides a simple quality control mechanism.

A 10 turn coil has been wound with BSCCO 2212 cable at BNL. To minimize the possibility of damage it was carefully wound by hand. Two such coils will be made and tested separately and in a common coil configuration. They will then be put in the background field of another common coil magnet.

The latest results [13] indicate that a $0.8 \mathrm{~mm}$ diameter BSCCO 2212 wire now carries over $600 \mathrm{~A}$ at $4.3 \mathrm{~K}$ in no background field. A field of $\sim 15 \mathrm{~T}$ would be created when an inner coil using the 18 strand cable made with such wire is used together with the $\mathrm{Nb}_{3} \mathrm{Sn}$ outer coils now under design for the high field magnet program. 


\section{CABle ReAction and CABle Test Facility At BNL}

BNL has a large furnace $\left(\sim 1.5 \mathrm{~m}^{3}\right)$ for reacting $\mathrm{Nb}_{3} \mathrm{Sn}$ cable in quantities sufficient to make full size magnets. Recently chrome-plated cables and non-chrome plated cables have been reacted. The cable that was not chrome-plated had to be treated with $100 \%$ Mobil- 1 oil to reduce sintering. A sample of the chrome-plated $\mathrm{Nb}_{3} \mathrm{Sn}$ cable was tested at the BNL's cable test facility. The results of these measurements are shown in Fig. 6 .

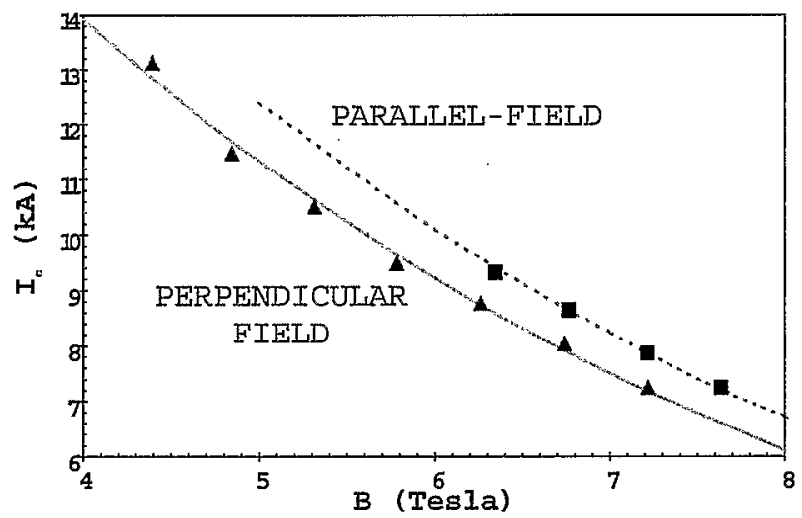

Fig. 6. Measurement of the field dependence of the critical current in $\mathrm{Nb}_{3} \mathrm{Sn}$ cable.

\section{Field Quality IN COMmon CoIl Design}

One of the early concerns about the common coil design was its ability to produce the good field quality required in accelerator magnets. In particular, the magnet ends are inherently top-bottom asymmetric [1-3]. It has been shown [14] that with proper choice of end spacers, the field harmonics can be theoretically reduced to a very small level with the code ROXIE [15]. In that model, the contribution of the ends to the integrated field errors in a $40 \mathrm{~mm}$ aperture, 15 meter long magnet was only $1-2$ parts in $10^{6}$ at $10 \mathrm{~mm}$ reference radius.

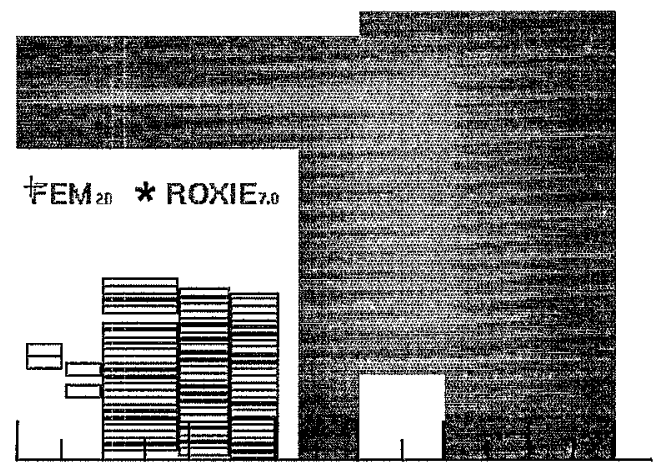

\section{$\begin{array}{llllllll}0 & 20 & 40 & 60 & 80 & 100 & 120 & 140\end{array}$}

Fig. 7: ROXIE model of the geometry optimized for field quality.

A magnetic model with minimized field harmonics in the cross-section is shown in Fig. 7. The design is based on flat racetrack coils with details presented elsewhere [16]. The

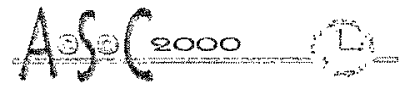

optimized harmonics are shown in Fig. 8. All geometric harmonics, including higher order terms, are only about a part in $10^{5}$ or less. The saturation-induced harmonics are also optimized to a level of 1 part in $10^{4}$ or smaller. The persistent current-induced harmonics are not discussed here.
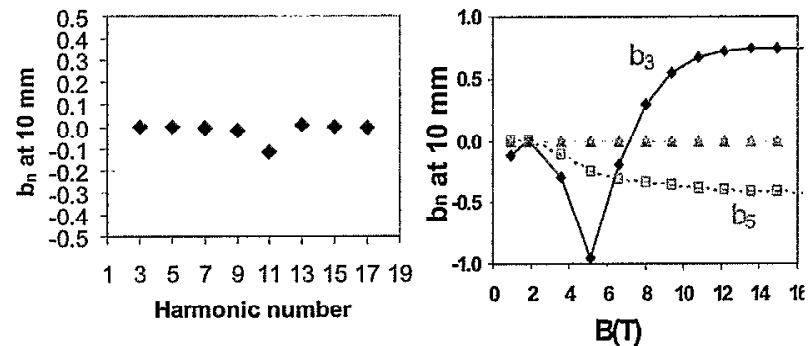

Fig. 8. Geometric (left) and saturation induced (right) harmonics in the units of $10^{-4}$ in a common coil magnet design.

\section{ACKNOWLEDGMENT}

We acknowledge the contribution of our technicians and designers and their enthusiasm for working on this new technology. In particular we thank Edward Weigand, Daniel Sullivan and Francis Skidmore for suggesting and implementing several good practical ideas. Collaborations with R. Scanlan of LBNL, R. Sokolowsky and L. Motowidlo of IGC and T. Hasegawa of Showa Electric are also appreciated.

\section{REFERENCES}

[1] http://magnets.rhic.bnl.gov/gupta/commoncoil.

[2] R. Gupta, "A Common Coil Design for High Field 2-in-1 Accelerator Magnets," Proceedings of the 1997 Particle Accelerator Conference.

[3] R. Gupta, et al., "A High Field Magnet Design for a Future Hadron Collider," Proceedings of the 1998 Applied Superconductivity Conference, Palm Desert, CA, Sept. 1998.

[4] R. Gupta, "Common Coil Magnet System for VLHC," Proceedings of the 1999 Particle Accelerator Conference.

[5] A. Ghosh, et al., "Common Magnet for Testing High Field Superconductors," Proceedings of the 1999 Particle Accelerator Conference.

[6] S. Gourlay, et al., "Fabrication and Test Results of a Prototype, $\mathrm{Nb}_{3} \mathrm{Sn}$ Superconducting Racetrack Dipole Magnet," Proceedings of the 1999 Particle Accelerator Conference.

[7] S. Gourlay, et al., "Fabrication and Test of $\mathrm{Nb}_{3} \mathrm{Sn}$ Racetrack Coils at High Field," This Conference.

[8] G. Ambrosio, et al., "Development of a React \& Wind Common Coil Dipole for VLHC," This Conference.

[9] A. den Ouden, private communication and University of Twente Internal Report UT-LBNL 99-01.

[10] CTD101 is a trademark of Composite Technology Development, Inc.

[11] Stycast is a trademark of Emerson \& Cuming Specialty Polymers.

[12] W. Sampson, et al., "Persistent Current effects in BSSCO Common Coil Dipole," This Conference.

[13] Y. Aoki; et al., "Improvement of Superconducting Properties of Bi2212 Round Wire and Primary Test Results of Large Capacity Rutherford Cable," This Conference.

[14] R. Gupta, et al., "Field Quality Optimization in a Common Coil Magnet Design," International Conference on Magnet Technology (MT-16) at Ponte Vedra Beach, Florida, USA (1999).

[15] S. Russenschuck, "ROXIE - A Computer Code for the Integrated Design of Accelerator Magnets," Sixth European Particle Accelerator Conference, Stockholm, Sweden, June 1998.

[16] R. Gupta, "Possible Future of RD3 As A Magnet R\&D Test Factory", LBNL Internal Note No. SC-MAG 697, unpublished. 